\title{
LA EDUCACIÓN COMO MECANISMO IGUALADOR DE OPORTUNIDADES: REEDITANDO VIEJAS DUDAS DESDE URUGUAY
}

\author{
Por Pablo López Gómez* \\ FLACSO-UDELAR, Uruguay \\ plopez@psico.edu.uy
}

Recibido: 30/09/2016 Aceptado: 22/11/2016

\begin{abstract}
Resumen
Los efectos de la educación sobre la igualdad ha sido un tema de discusión permanente desde los años 50. Por un lado, se ha intentado corroborar un efecto positivo en la distribución más equitativa de los ingresos y en el desarrollo de los países (principalmente a través de la teoría del Capital Humano), mientras que, por otra parte, se señala su presunto efecto sobre la posibilidad de acceder de forma más equitativa a los beneficios sociales a través la igualación de oportunidades. En la actualidad, este segundo aspecto resulta el más señalado cuando se promueven políticas educativas tendientes a la universalización de secundaria. El presente artículo busca discutir acerca de las posibilidades de la educación de conseguir la anhelada igualdad de oportunidades a través del análisis de bases de datos internacionales y uruguayas, controlando dos factores considerados exógenos al individuo (género y etnia). El análisis revela que, contando con los mismos años de educación, estos fac-
\end{abstract}

* Doctor en Educación en la UNER (Paraná), Licenciado en Educación (UTEM - Santiago de Chile) y Educador Social (CENFORES - Montevideo), Diplomado en Ciencias Sociales con Mención en Género y Políticas (FLACSO - Buenos Aires) y Diplomado en Análisis de Información Sociodemográfica aplicada a la gestión (FCS - UDELAR - Montevideo). Profesor Adjunto en el Instituto de Psicología de la Salud (Facultad de Psicología, UDELAR), Coordinador alterno del Programa Género, Salud Reproductiva y sexualidades. Profesor de la Maestría en Género Sociedad y Políticas (FLACSO Argentina). 
tores exógenos suelen pesar más que la paridad educativa en variables como desempleo e ingresos. Luego se propone una discusión teórica a propósito de las limitaciones de la educación para lograr la igualdad de oportunidades, y se esbozan las posibles explicaciones de esta limitación.

Palabras Clave: Educación - Secundaria -Igualdad de oportunidades -Género - Etnia

\section{Abstract}

Since the 1950 the effects of education on equality has been an ongoing subject of discussion. On one hand, it has tried to corroborate a positive impact on more equitable distribution of income and development of the countries (mainly through the theory of Human Capital), while on the other hand, it is alleged the effect on the possibility of more equitable access to social benefits through the equalization of opportunities. Nowadays, this second aspect is more incisive when tending to promote the universalization of secondary education policies. This article seeks to discuss the possibilities of education to achieve the desired equal opportunities through analysis of international and uruguayan data bases, controlling two factors considered exogenous to the individual (gender and ethnicity). The analysis revealed that, with the same years of education, these exogenous factors often outweigh educational parity on variables such as unemployment and income. Through empirical evidence proposed a theoretical discussion about the limitations of education in order to achieve equal opportunities, outlining possible explanations for this limitation.

Keywords: Education - Highschool - Equality of Opportunity - Gender - Ethnicity

\section{Introducción}

El presente artículo resume uno de los capítulos de la tesis de doctorado ¿Por qué universalizar la secundaria? Dudas razonables acerca de las respuestas políticas y técnicas habituales, dirigida por el Profesor Emérito Dr. Christian Baudelot y defendida en diciembre de 2015. La tesis, luego de una amplia recopilación de datos uruguayos, regionales y mundiales acerca de la interrupción de las trayectorias educativas de los adolescentes en secundaria, se interroga acerca de cuál es el origen de la preocupación de los gobiernos por la universalización del nivel. Para ello se revisaron 32 documentos oficiales y técnicos (10 uruguayos y 22 elaborados por agencias y organismos internacionales que refieren entre otros países a Uruguay), tomando como criterio de inclusión que tuvieran hasta 15 años e hicieran recomendaciones tendientes a la universalización de la secundaria. Se analizaron los fundamen- 
tos de estas recomendaciones, encontrándose dos grupos de motivaciones: a) uno refería al desarrollo de competencias para la inserción social y; b) otro señalaba los efectos de la educación para el logro de objetivos como la movilidad social, igualdad y equidad.

Luego de ese primer análisis, se tomó la decisión de centrar la tesis en los argumentos que vinculan a la educación con la movilidad social, el desarrollo, la igualdad y la equidad. Se dividió el análisis en tres capítulos: uno que analiza el efecto de la educación en la distribución de los ingresos y el desarrollo, poniendo en duda los postulados de la teoría del capital humano; otro que analiza la relación entre educación e igualdad de oportunidades que parcialmente se presenta aquí (discutiendo especialmente con la teoría de Roemers (1998) que es la versión de esta teoría de justicia más extendida actualmente); y un tercer capítulo que discute algunas versiones de la teoría del capital social (Coleman, 1998; Fukushama, 1997; Woolkok, 1998), que centra sus explicaciones acerca de las limitaciones del sistema educativo para el logro de la igualdad en la cuestión de la calidad.

Como se plantea desde el título, la tesis -y también este artículo- no pretende desacreditar toda la evidencia empírica que marcan ciertas correlaciones positivas entre nivel educativo y bienestar social (a nivel individual y colectivo). Simplemente busca destacar la otra cara de la moneda, mostrando datos que permiten afirmar que esas correlaciones ni son universales, ni están por fuera de una coyuntura determinada. La constatación de una invisibilización de los límites de los efectos de la educación -que se lee en los documentos oficiales y técnicos revisados- motivó la actualización de algunos de los postulados que, oportunamente, señalaran los teóricos de la reproducción. Hay evidencia empírica que permite señalar la vigencia de muchos de las comprobaciones que se hicieron en los años 70 y 80 , a pesar de los esfuerzos de los gobiernos por implementar reformas en base a las recomendaciones de las teorías con las que se discute.

Este artículo resume parte del capítulo sobre desigualdad de oportunidades y se organiza de la siguiente manera: a) primero se plantea brevemente cuáles son los postulados de la teoría de la igualdad de oportunidades y el papel que la educación jugaría en su consecución; b) luego se presenta evidencia empírica tomada de estudios internacionales y de bases de datos abiertas que señalan cómo, a pesar del efecto de la educación, dos categorías productoras de desigualdad siguen teniendo efecto en el logro de los personas (etnia, género); c) luego se introducen brevemente algunas críticas actuales a las teorías de justicia basadas en la igualdad de oportunidades; y d) finalmente se esbozan algunas conclusiones. En la tesis el capítulo analiza también el efecto de la educación sobre la movilidad social y toma como otro factor exógeno a analizar el nivel socio-económico de la familia de origen. No obstante, dado la extensión de ese análisis, no se incluyen en este artículo, 
aunque es de recibo comentar que las conclusiones del mismo son similares a las presentadas respecto a género y etnia.

\section{Definiendo la igualdad de oportunidades}

Tanto en las producciones nacionales, como en los documentos de las agencias revisados, la idea más repetida en referencia a la igualdad es la de "igualdad de oportunidades". En algunos casos se contrapone de forma explícita a la idea de "igualdad de resultados", en general y a una distribución menos concentrada de los ingresos, en particular. En un artículo de la revista del FMI, al respecto de la igualdad de oportunidades, se señala que este enfoque es heredero de los aportes de "John Rawls, Ronald Dworkin, Amartya Sen y John Roemer" (Ferreira y Walton, 2005, p. 1). Si bien es cierto que hay diferencias (en algunos casos substanciales) entre estos autores, no es menos cierto que de alguna manera todos buscaban la respuesta a una pregunta que se impuso desde los años 70: ¿qué desigualdades pueden considerarse justas y cuáles desigualdades son injustas?, es decir, cuál es la base normativa de la igualdad. Dado que el citado artículo del FMI evoca la noción de igualdad de oportunidades postulada por el Banco Mundial, en su informe "Equidad y desarrollo" de 2006, es de recibo retomar los posicionamientos explícitos del organismo a propósito de esta cuestión.

En 2008 el Banco Mundial publica el informe "Midiendo la Desigualdad de Oportunidades en América Latina y el Caribe". En él se comenta que, el surgimiento de la igualdad de oportunidades como horizonte de justicia, aparece como un intento por superar las medidas de igualdad basadas, sobre todo, en la distribución de la renta. Los autores señalan que, dado que los hombres somos diversos, resulta natural que obtengamos resultados diversos. La cuestión se planteaba en estos términos:

La distinción primordial entre las diferencias de resultados que son atribuibles a la responsabilidad individual y aquellos que no los son jugó un papel central en la filosofía política de los últimos treinta a cuarenta años. Antes del libro de John Rawls, A Theory of Justice (1971), la mayoría de las personas intentaban evaluar la justicia o la equidad social solamente sobre la base de la distribución de los resultados. En los años '70, estimulados por los trabajos de Rawls y de Robert Nozick (1974), los científicos y filósofos políticos comenzaron a considerar la equidad de los procesos, y cómo los resultados finales están determinados tanto por las oportunidades de las que goza una persona, como por lo que esa persona hace con ellas. (Paes de Barros et al, 2008, p. 47).

Lo que uniría a autores como Rawls (1995), Sen (2000), Dworkin (2000), Roemer (1998) o Arneson (1989) es la idea de que es necesario definir una base normativa para determinar, cuándo una distribución de resultados es "justa" y cuándo no lo es. Se trataría de una intuición acerca del hecho de que 
Revista de la Escuela de Ciencias de la Educación, año 13, nRo. 12, vol. 1, enero a Junio de 2017. PÁGINAS 151-173. ISSN 1851-6297 - ISSN 2362-3349 (EN LINEA) LA EDUCACIÓN COMO MECANISMO IGUALADOR DE OPORTUNIDADES: REEDITANDO VIEJAS DUdAS DESDE URUguay. PABLo LóPEZ Gómez

la justicia requería "equidad de algo", pero esa "equidad" debía reconocer el peso del esfuerzo individual.

Se desconoce cuáles son las fuentes normativas del concepto de "igualdad de oportunidades" que postula cada agencia y cada producción uruguaya relevada, pero lo cierto es que hay diferencias entre los autores mencionados por el Banco Mundial, y las mismas dan por resultado distintas formas de entender la igualdad y diferentes formas de medirla. El concepto de "igualdad de oportunidades" propiamente dicho es heredero de la teoría de la justicia de Rawls (1995), y más modernamente de los desarrollos de Roemer (1998). Dworkin (2000) elaboró la idea de "igualdad de recursos", Arneson (1989) de "igualdad de oportunidades para el bienestar", y Sen (2000) introdujo el "enfoque de capacidades". No corresponde entrar en detalle acerca de lo que postula cada una de estas teorías, pero si señalar que, aun teniendo en común el hecho de que su noción de justicia distributiva combina elementos sociales y personales, los elementos tomados en cuenta en una y otra teoría difieren.

Uno de los trabajos que realizan un intento de elaborar un índice de igualdad de oportunidades para América Latina es el de Paes de Barros et al. (2008), y es importante retomarlo puesto que tres de sus autores trabajan en el Banco Mundial. Este organismo, además, editó el libro y es uno de los organismos que ha postulado la importancia de la educación para la igualdad de oportunidades en la región. En este libro se trabaja sobre la noción normativa de igualdad de oportunidades basada en Roemer.

Una definición útil para esta discusión es la de Roemer (1998). Roemer habló de las variables de resultado (el ingreso, el logro educativo, etc.) como una "ventaja", y dividió los determinantes de la ventaja en dos grupos: "esfuerzos" - que dependen de las preferencias de la persona- y las "circunstancias", que son los factores que caen fuera del control de la persona. La igualdad de oportunidades prevalecería en una situación en donde la distribución de un resultado de interés es independiente de las circunstancias. La igualdad de oportunidades nivela el terreno de juego y todos tienen, en principio, el potencial de alcanzar los resultados de su elección (Paes de Barros et al, 2008, p. 48).

La noción de igualdad de oportunidades de Roemer (1998) busca superar algunas dificultades que, para el autor, tienen otras formas de concebir la idea. Sobre este punto puede resultar esclarecedora la clasificación realizada por Puyol (2010), a propósito de las formas de entender la desigualdad de oportunidades. La misma establece que se puede dividir las formas de entender la igualdad de oportunidades, en primer término, en dos grandes posiciones al respecto del alcance del término: a) llama a la primera igualdad formal de oportunidades y se reduciría a la idea de garantizar legalmente que nadie será discriminado en el acceso a los bienes sociales por una condición deter- 
minada (raza, sexo, origen social, etc.); b) denomina a la segunda corriente, igualdad equitativa de oportunidades, y estaría caracterizada por postular que las diferencias socioeconómicas impiden que la igualdad legal sea suficiente, y plantean principalmente una nivelación de esa desigualdad a través de la educación. Para ellos, el talento debe ser el diferencial que explique la desigualdad de resultados y no otra circunstancia fuera del control de las personas. El carácter "equitativo" de esta concepción le otorga la necesidad de distribuir los esfuerzos comunes, de forma de beneficiar a los que parten de situaciones más desventajosas.

Luego establece otras dos posiciones respecto al tipo de desigualdad de resultado que la igualdad de oportunidades debe generar: a) la concepción social de la igualdad de oportunidades, que considera que lo que es inadmisible es que se pueda predecir, desde el nacimiento, la posición que ocupará un sujeto debido a sus condicionantes. Esta corriente confía que las políticas sociales, sobre todo las políticas educativas corrijan la desigualdad de oportunidades haciendo "legitima", la desigualdad de resultados. Se trata de la idea que postula la legitimidad moral de la meritocracia (quienes son mejores llegan a las posiciones más recompensadas); b) la concepción individualista de la igualdad de oportunidades, centra su mirada en el resultado personal y no grupal, considerando que habrá diferencias entre los individuos en las mismas circunstancias que derivan de su esfuerzo individual. En la igualdad de oportunidades social, dos personas en las mismas condiciones en términos de "oportunidades sociales", pueden tener resultados muy diversos dependiendo de su esfuerzo y talento. Para la concepción individualista, el esfuerzo es un diferencial moralmente justo, pero no lo es talento porque, que se cultive o no el mismo, puede depender de las circunstancias.

Puyol (2010) coloca a Roemer (1998) en esta última categoría. Retomando directamente el trabajo de Roemer se observa que el autor plantea:

El objetivo de una política de igualdad de oportunidades es asignar recursos de modo que los resultados que una persona obtenga se correspondan solamente con su esfuerzo y no con sus circunstancias. Puesto que hemos propuesto como criterio de comparación intertípica del esfuerzo el centil de su distribución, la política que propongo es aquella que ofrezca resultados -en este caso, la capacidad para ganarse la vida en un futuro- tan iguales como sea posible entre aquellos individuos de distintos tipos situados en un mismo centil de sus respectivas distribuciones de esfuerzos. En cualquier caso, entre los individuos de cada tipo se pueden dar grandes diferencias respecto a su capacidad de ganarse la vida según varíe su esfuerzo. (1998, p. 77).

Para comprender la referencia es importante aclarar algunas ideas previas que desarrolla el autor: a) su ejemplo se remitía a las cuestiones educativas, 
Revista de la Escuela de Ciencias de la Educación, año 13, nRo. 12, vol. 1, enero a Junio de 2017. PÁGINAS 151-173. ISSN 1851-6297 - ISSN 2362-3349 (EN LíneA) La EdUCACIÓN COMO MECANISMO IGUALADOR DE OPORTUNIDADES: REEDITANDO VIEJAS DUdAS DESDE URUguay. PABLo LóPEZ Gómez

suponiendo que tengo un presupuesto para dividir entre las escuelas; b) la referencia al centil de distribución se comprende en el marco de su propuesta operativa. La misma consiste en agrupar a los niños en determinados "tipos", donde las circunstancias sean lo más similares posibles. Evidentemente la propuesta es complejísima de operacionalizar para su medición (aunque el autor propone un algoritmo para lograrlo) y requiere de una serie de decisiones que son ético-políticas. El propio Roemer lo explicita de forma muy clara:

Entre la ciudadanía de cualquier democracia avanzada, encontramos individuos con opiniones muy diversas sobre lo que es necesario para la igualdad de oportunidades, desde la concepción no discriminatoria, en un extremo, hasta la intervención social para corregir todo género de desigualdades, en el otro. En cualquier caso, es común a todas ellas el precepto de que el principio de igualdad de oportunidades exige en algún momento que el individuo se haga responsable de la consecución de tal igualdad, bien se refiera ésta a una determinada cualificación escolar, salud, nivel de empleo o salario, o a la utilidad o bienestar del economista. Por tanto, hay un «antes» y un «después» en el concepto de igualdad de oportunidades: antes de que comience la competición deben igualarse las oportunidades, incluso mediante una intervención social, si es necesario; pero una vez que comienza, los individuos han de asumir plenamente su responsabilidad. Pueden clasificarse las distintas concepciones de la igualdad de oportunidades de acuerdo con el lugar en el que sitúen el umbral a partir del cual los individuos asumen esta responsabilidad. (Roemer, 1998, p. 72).

Es decir, aun cuando se dice, como en el caso de trabajo de Paes de Barros et al (2008), que es útil la definición de igualdad de oportunidades de Roemer (1998), será necesario además explicitar que se considera desigualdad de oportunidades. Es decir, hay que determinar cuáles son las circunstancias que se consideran injustas y a partir de que umbral se comienza a considerar que el resultado desigual es fruto del esfuerzo de la persona y no de sus circunstancias.

Si bien este desarrollo surge del posicionamiento del Banco Mundial sobre la cuestión de la desigualdad de oportunidades, cabría preguntarse si la posición de la CEPAL es la misma. En principio, retomando la clasificación de Puyol parecería que esta agencia se coloca en una postura más vinculada a la concepción social de la igualdad de oportunidades y apunta a demostrar la diferencia en los resultados entre grupos sociales y proponer soluciones para disminuirlas, sin detenerse a analizar los esfuerzos individuales de cada integrante de esos grupos. Asimismo, no hace una distinción entre talento y esfuerzo y propugna por una sociedad más meritocrática (CEPAL, 2007).

En los hechos tanto la CEPAL como el Banco Mundial, al intentar demostrar empíricamente la desigualdad de oportunidades y, fundamentalmente, el 
efecto de la educación para el logro de la misma, se colocan desde lo que se ha llamado concepción social de la igualdad de oportunidades.

Aún en el informe sobre la desigualdad de oportunidades de Paes de Barros et. Al (2008), luego de utilizar la definición de Roemer (1998), se operacionaliza un índice de desigualdad de oportunidades que sirve para indicar la diferencia entre los grupos, pero no distingue el nivel de esfuerzos de las personas para el caso de los niños.

En la medición de la desigualdad de oportunidades, para poder observar las diferencias en los beneficios que obtienen distintos grupos, los documentos revisados acuden a indicadores de resultado clásicos como el ingreso, el acceso al trabajo y distintas posiciones de poder o la posibilidad de disfrutar de determinados bienes sociales. Aquí se seguirá este mismo procedimiento para las variables etnia y género.

La teoría del capital humano retoma dos perspectivas de justicia: por un lado, se busca igualar las oportunidades y por otro se espera un efecto conjunto de disminución de la dispersión salarial. Este efecto, cabe aclarar, sería un resultado de la mayor equidad y del aumento conjunto de la productividad de la población. No se trataría de un objetivo de justicia sino de un resultado, podría decirse, colateral. La afinidad central entre la teoría de justicia basadas en la igualdad de oportunidades y la teoría del capital humano estaría en el poder de la educación para "nivelar el campo de juego" en palabras de Roemer (1998).

En este artículo se revisa el efecto de la educación en la generación de igualdad de oportunidades, centrado la mirada en dos afirmaciones: a) la educación debiera actuar como nivelador, permitiendo el desarrollo del potencial del individuo disminuyendo o anulando el efecto de factores exógenos que podrían generar desigualdad; b) desde el punto de vista de la teoría del capital humano la inversión personal en educación es rentable y, por tanto, mayores niveles educativos aumentan las probabilidades de acceso a beneficios sociales. Este reparto, en base al nivel educativo, sería justo puesto que implica que quienes logran los beneficios se esforzaron para alcanzarlos a través de su propia educación. Para que estas afirmaciones funcionen, la educación debería tener una fuerte correspondencia con el logro individual, con independencia de los factores exógenos que se analizarán (género y etnia).

Estas dos discusiones implican la revisión de indicadores complejos que no siempre están disponibles en la misma base de datos, lo cual supone limitaciones al ejercicio de comparación internacional. Aún con esta limitación se encontró suficiente información comparable como para poder aproximar algunas conclusiones, que serán más precisas para el caso específico de Uruguay. 
Revista de la Escuela de Ciencias de la Educación, año 13, nRo. 12, vol. 1, enero a junio de 2017. PÁGINAS 151-173. ISSN 1851-6297 - ISSN 2362-3349 (EN LíneA) La EdUCACIÓN COMO MECANISMO IGUALADOR DE OPORTUNIDADES: REEDITANDO VIEJAS DUdAS DESDE URUguay. PABLo LóPEZ Gómez

\section{Género e igualdad de oportunidades}

Si bien se constata un avance importante de las mujeres en su búsqueda de igualdad respecto de los hombres desde mediados del siglo XX, en todos los países del mundo persisten, al día de hoy, importantes brechas en los logros de las mujeres. No corresponde aquí profundizar en un tema tan complejo como el análisis teórico de la desigualdad de género a nivel mundial, pero será necesario presentar indicadores vinculados al mercado de trabajo e ingresos, que son aquellos que permiten estimar si la educación produce el efecto que prevé la teoría del capital humano y la igualdad de oportunidades.

Un indicador muy relevante es la tasa de actividad, puesto que la división público - privado establecía un régimen donde las mujeres se ocupaban de la reproducción social (crianza y educación de los niños y tareas domésticas), y los hombres de la producción. Esto implicó que las mujeres estuvieran mayoritariamente fuera del mercado de trabajo remunerado, situación que empieza a revertirse con fuerza desde los 60 , pero que aún conserva importantes brechas como se ve en la gráfica 1.

Se observa también, que la participación de las mujeres en el mercado de trabajo es menor en todos los países de la región, con una brecha de 25 puntos porcentuales en el promedio. La brecha más chica de la región corresponde a Uruguay, con 17 puntos, y la más importante a Venezuela que prácticamente la duplica $(33,5 \%)$.

\section{Gráfica 1: Tasa de actividad por sexo. América Latina 2011 o último dato disponible.}

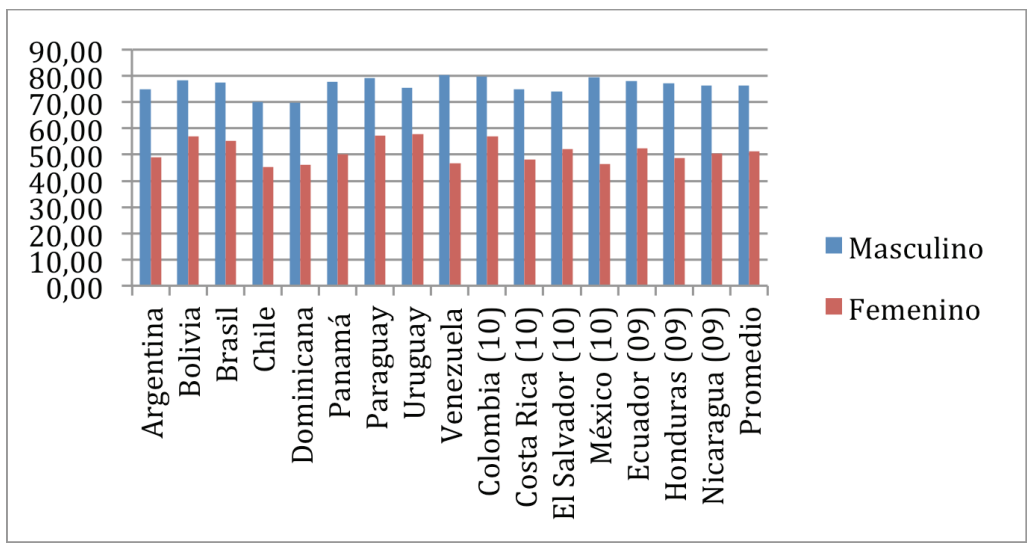

Fuente: elaboración propia en base a Siteal (consultado en 2013) 
La pregunta acerca de cómo la educación afecta este indicador tiene, a diferencia de otros indicadores revisados, una respuesta clara: la acumulación de años de estudio afecta positivamente la posibilidad de las mujeres de participar en el mercado de trabajo. Las siguientes gráficas muestran esta tendencia para los países de la subregión, pero la misma pauta puede observarse en todos los países seleccionados. La tasa de participación de los varones sube y se estabiliza hasta el período 10 a 12 años de estudio, mientras que la de las mujeres sigue subiendo y comienza a cerrar la brecha.

Gráfica 2: Tasa de actividad por sexo y años de estudio. Cono Sur (Argentina, Brasil, Chile y Uruguay).

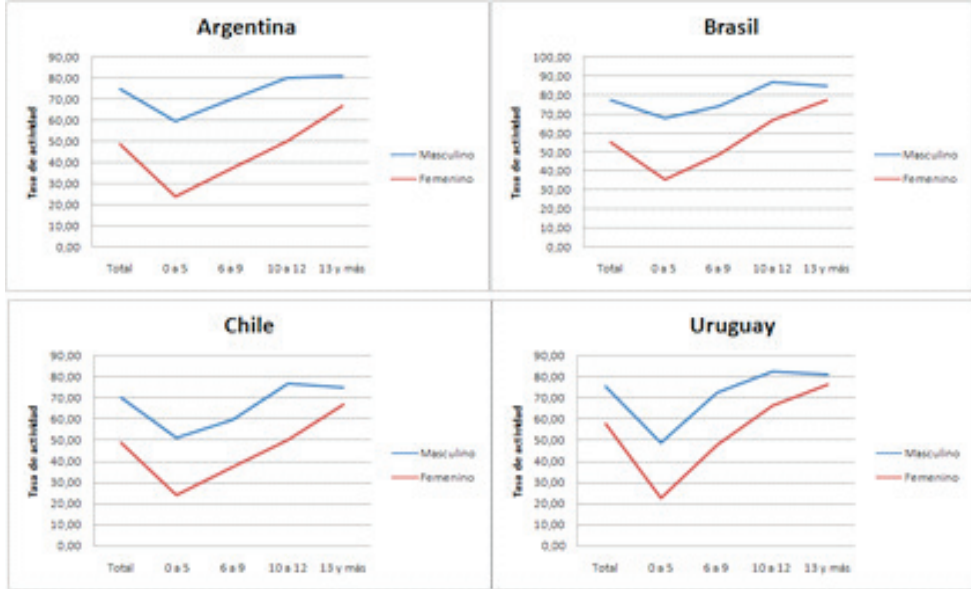

Fuente: elaboración propia en base a Siteal (consultado en 2013).

En el total, la brecha más grande es la de Argentina con 26 puntos, y las más pequeña la de Uruguay con 17, teniendo Chile 24 y Brasil 22. Con 13 o más años de estudio la brecha de Argentina se cierra hasta un $14 \%$ y la chilena a $12 \%$, la uruguaya llega apenas a un $4 \%$ y la de Brasil a un $7 \%$.

Si bien es esperable que, la participación de la población en general en el mercado de trabajo aumente a medida que aumentan los años de estudio, el hecho de que para las mujeres ese aumento sea mayor que para los varones estaría indicando que podría haber un efecto de la educación sobre esta este indicador, emparejando las diferencias por sexo.

No obstante, es un poco difícil estimar el peso de la variable años de estudio exclusivamente, entre otras cosas por su fuerte correlación con el ingreso familiar y clima educativo del hogar. Si bien es cierto que esta correlación afecta tanto a varones como mujeres (es decir, suelen tener mayores ingresos 
quienes tienen más educación), también es cierto que para que los ingresos laborales valgan la pena normalmente las familias suelen considerar el costo que les representa solucionar las necesidades de los niños (cuidado, alimentación, etc.), cuando los dos padres trabajan. Por las características del "contrato sexual" (tomando el término de Pateman, 1995), cuando el salario percibido por las mujeres no compensa los gastos que produce la crianza, muchas se inclinan por permanecer en la casa y por tanto no integran la población económicamente activa. Esto podría condicionar lo visto en la gráfica de la siguiente manera: las mujeres con más educación perciben mayores ingresos y por tanto se integran al mercado de trabajo (porque les es redituable), las que tienen menos educación no se integran porque los ingresos que percibirán no compensan los gastos que genera su inserción. Para los varones, en general, este condicionamiento les es indiferente. Eso implicaría que la educación no cambia la posición social de las mujeres, sino que simplemente les permite acceder a facilidades (a través de un mayor ingreso), que les permite acercarse al desempeño de los varones en el indicador tasa de actividad. Si bien el efecto no sería directo, tampoco puede desconocerse que estaría afectando en algo el acceso a las oportunidades.

Es factible además que haya otro efecto de la educación que influya en este indicador: el número de hijos. En América Latina hay una fuerte correspondencia entre los años de educación de las mujeres y el número de hijos que tiene, a mayor cantidad de años de estudio, es menor el número de hijos. También hay una correspondencia particularmente intensa entre el número de hijos de una mujer y su posibilidad de formar parte de la población económicamente activa. Trascendería el tema del artículo presentar los datos de todos los países, que no son parte de los indicadores habitualmente comparados en las bases de datos. Pero es pertinente mostrar cómo funciona esta relación en Uruguay. El siguiente cuadro forma parte del trabajo de Varela et al (2008). En él puede observarse la paridez media acumulada de distintos grupos de edad para el año 1996 y 2006. Si se toma el grupo de mujeres que ya han cerrado su ciclo reproductivo se observa que para el 2006, aquellas mujeres con primaria incompleta y con el ciclo básico incompleto tenían un promedio de más de tres hijos. En el otro extremo, las mujeres con bachillerato y las que tienen estudios universitarios completos no llegan a 2 hijos por mujer. Si se observan además los grupos típicos del momento de inserción laboral (de 20 a 24), vemos que las mujeres con menor educación (primaria incompleta y ciclo básico incompleto) tienen una paridez acumulada de alrededor de $1(1,14$ y 1,02).

Es decir en promedio ya tienen un hijo. Las mujeres más educadas (bachillerato y más) apenas tienen un 0,1. Es decir, cada 10 mujeres de 20 a 25 años con baja educación que tiene hijos sólo 1 con mayores logros educativos los tiene. 
Revista de la Escuela de Ciencias de la Educación, año 13, nRo. 12, vol. 1, enero a junio de 2017. PÁGINAS 151-173. ISSN 1851-6297 - ISSN 2362-3349 (EN LINEA) LA EDUCACIÓN COMO MECANISMO IGUALADOR DE OPORTUNIDADES: REEDITANDO VIEJAS DUdAS DESDE URUguay. Pablo López Gómez

Cuadro 1: Paridez media por nivel educativo según grupo de edad, Uruguay, 1996 -2006.

\begin{tabular}{|c|c|c|c|c|c|c|c|c|c|c|}
\hline & \multicolumn{2}{|c|}{ Primaria incompleta } & \multicolumn{2}{|c|}{ Primaria y ciclo básico incompletd } & \multicolumn{2}{|c|}{ Ciclo básico Bachillerato inc } & \multicolumn{2}{|c|}{ Bachillerato Universidad incompleta } & \multicolumn{2}{|c|}{ Universitario complett } \\
\hline & 1996 & 2006 & 1996 & 2006 & 1996 & 2005 & 1996 & 2006 & 1996 & 2006 \\
\hline 15 a 19 & 0,46 & 0,22 & 0,26 & 0,21 & 0,08 & 0,04 & $x$ & $x$ & $x$ & $x$ \\
\hline $20 \mathrm{a} 24$ & 1,43 & 1,14 & 1,02 & 1,02 & 0,47 & 0,52 & 0,16 & 0,1 & 0,16 & 0,11 \\
\hline 25 a 29 & 2,27 & 2,19 & 1,74 & 1,83 & 1,06 & 1,19 & 0,6 & 0,37 & 0,44 & 0,34 \\
\hline $30 \mathrm{a} 34$ & 2,88 & 2,54 & 2,32 & 2,48 & 1,71 & 1,74 & 1,32 & 1,01 & 1,08 & 0,99 \\
\hline 35 a 39 & 3,36 & 3,29 & 2,73 & 2,84 & 2,1 & 2,1 & 1,8 & 1,57 & 1,64 & 1,43 \\
\hline $40 \mathrm{a} 44$ & 3,59 & 3,59 & 2,92 & 3 & 2,24 & 2,31 & 2,01 & 1,21 & 1,88 & 1,75 \\
\hline $45 \mathrm{a} 49$ & 3,59 & 3,61 & 2,85 & 3,04 & 2,26 & 2,39 & 2,04 & 1,95 & 2,01 & 1,83 \\
\hline
\end{tabular}

Fuente: Varela, Pollelo y Fostik, p. 37.

Si se sospecha que hay una fuerte correlación entre ingresos y nivel educativo cabría, por lo menos instalar la duda, acerca de cuál es el peso de cada variable en la paridez. Incluso más, cabe preguntarse si la paridez es una variable dependiente del nivel educativo, o más bien ambas variables son simplemente dos consecuencias de la desigualdad social, entendida no sólo desde la perspectiva de los ingresos, sino como una cuestión de "ethos de clase" en palabras de Bourdieu. En López y Varela (2014) se plantea, para Uruguay, que el $70 \%$ de las adolescentes madres interrumpieron sus estudios antes de quedar embarazadas. El nivel educativo alcanzado, así como la paridez media, podrían depender precisamente de unas condiciones de vida y una forma de entenderla, valorarla y vivirla que la educación que efectivamente se ha universalizado, la primaria, no logra afectar.

La siguiente gráfica completa la asociación, mostrando el impacto de los hijos en la tasa de participación de las mujeres y hombres en la población económicamente activa. Ha sido extraído del informe sobre indicadores de género elaborado cada año por el Instituto Nacional de las Mujeres (p.27)

\section{Cuadro 2: Tasa de actividad de mujeres y varones (18 a 49 años) según presencia de menores de 13 años en el hogar.}

Cuadro 10. Tasa de actividad de mujeres y varones (entre 18 y 49 años) según presencia de menores de 13 años en el hogar. Total país, 2009 y 2013

\begin{tabular}{|c|ccc|ccc|}
\hline \multirow{2}{*}{$\begin{array}{c}\text { Tramos de } \\
\text { edad }\end{array}$} & \multicolumn{3}{|c|}{$\mathbf{2 0 0 9}$} & \multicolumn{3}{c|}{$\mathbf{2 0 1 3}$} \\
\cline { 2 - 7 } & Varones & Mujeres & Total & Varones & Mujeres & Total \\
\hline 0 & $88,6 \%$ & $78,0 \%$ & $83,5 \%$ & $89,0 \%$ & $78,6 \%$ & $84,2 \%$ \\
\hline 1 & $95,0 \%$ & $76,2 \%$ & $84,4 \%$ & $95,0 \%$ & $76,8 \%$ & $84,9 \%$ \\
\hline 2 & $97,4 \%$ & $71,9 \%$ & $83,3 \%$ & $96,9 \%$ & $71,1 \%$ & $82,7 \%$ \\
\hline 3 y más & $96,3 \%$ & $61,0 \%$ & $76,4 \%$ & $95,8 \%$ & $61,6 \%$ & $76,0 \%$ \\
\hline Total & $\mathbf{9 2 , 1} \%$ & $\mathbf{7 5 , 0} \%$ & $\mathbf{8 3 , 2} \%$ & $\mathbf{9 2 , 1} \%$ & $\mathbf{7 5 , 5 \%}$ & $\mathbf{8 3 , 6} \%$ \\
\hline
\end{tabular}

Fuente: La Buonora et al, 2013, p. 26. (Estadísticas de género Inmujeres.)

Como puede observarse, la posibilidad de formar parte de la población 
Revista de la Escuela de Ciencias de la Educación, año 13, nRo. 12, vol. 1, enero a junio de 2017. PÁGINAS 151-173. ISSN 1851-6297 - ISSN 2362-3349 (EN LINEA) LA EDUCACIÓN COMO MECANISMO IGUALADOR DE OPORTUNIDADES: REEDITANDO VIEJAS DUdAS DESDE URUguay. PABLo LóPEZ Gómez

económicamente activa disminuye para las mujeres a medida que tienen hijos, sobre todo cuando son pequeños. Para no caer en la complejidad del análisis (que no podría hacerse sin datos específicos) de cuánto es el efecto hijo y cuánto el efecto clase, ingreso, educación, etc., vale la pena observar el cambio en la brecha: sin hijos la brecha entre hombres y mujeres es del $10 \%$, con 3 o más hijos es del $30 \%$.

Finalmente, aún con todas las salvedades hechas al respecto de esta relación (años de estudio - tasa de actividad), es la única que muestra una línea clara. No sucederá lo mismo con los demás indicadores.

Si se considera el desempleo, es decir, las personas que forman parte de la población económicamente activa, pero que no consiguen trabajo, las brechas de género tienen una tendencia clara pero no unánime en la región. En general las mujeres tienen más dificultades para conseguir empleo como se observa en la siguiente gráfica.

Gráfica 3: Desempleo por sexo, países de América Latina, año 2011 o último dato disponible.

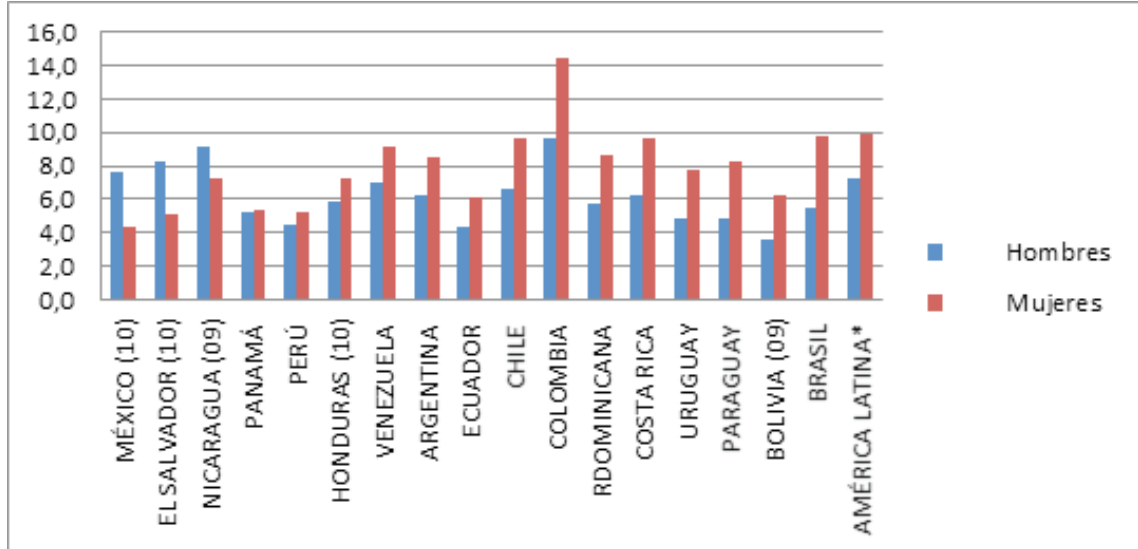

Fuente: elaboración propia en base a Siteal (consultado en 2013).

De los 17 países presentados, en 13 el desempleo femenino es mayor. La brecha más grande es la de Brasil donde la relación entre el desempleo femenino y el masculino alcanza al 1,8. Para Uruguay este valor es de 1,6. En México y El Salvador es de 0,6 y de 0,8 en Nicaragua. En Panamá habría paridad en el indicador.

La desocupación es uno de los indicadores que permiten observar con claridad el acceso a las oportunidades. A menudo se da por sentado que ser o no parte de la población económica activa es una decisión personal y no tanto una cuestión de oportunidades (aunque como se vio arriba, eso no siempre es 
así en el caso de las mujeres), lo cual no sucede con el desempleo, que implica una actitud activa que se ve limitada por circunstancias del mercado de trabajo. La brecha en la tasa de desempleo señala que hay más mujeres que varones que buscan activamente un empleo y señala la desigualdad de oportunidades entre unos y otras en la mayoría de los países de América Latina.

Corresponde entonces observar si la variable educación tiene un efecto igualador en esta estructura de oportunidades.

En la siguiente gráfica se observa la relación entre el desempleo femenino y masculino por años de estudio para los mismos países de América latina. Si hubiera una correspondencia afín a lo que plantea la teoría del capital humano, cabría esperar que a mayor educación la brecha en el desempleo tendiera a cerrarse. Como puede apreciarse esto no resulta así y los comportamientos de los países no guardan ninguna regularidad. Podría decirse que Chile y Ecuador tienen el comportamiento esperado mientras países como Paraguay, Honduras y Argentina tienen el comportamiento contrario, es decir a más educación por parte de las mujeres, más se agranda la brecha con los hombres de su mismo nivel.

\section{Gráfica 4: Ratio desempleo femenino sobre masculino por años de} estudio, países de América Latina, año 2011 o último dato disponible.

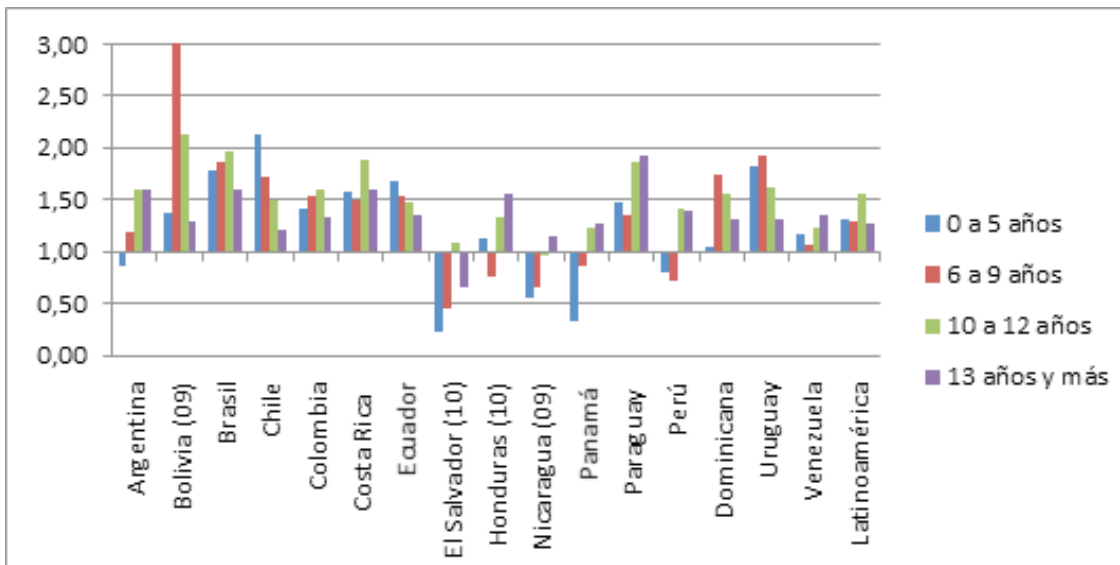

Fuente: elaboración propia en base a Siteal (consultado en 2013).

Uruguay tiene un comportamiento irregular. Las mujeres más educadas de los grupos analizados tienen una brecha en el desempleo menor. Sin embargo, no son las menos educadas, sino aquellas que están en una categoría intermedia (6 a 9 años) las que tienen una brecha mayor.

En Nicaragua la tendencia es exactamente la contraria a la esperada. Las 
Revista de la Escuela de Ciencias de la Educación, año 13, nRo. 12, vol. 1, enero a junio de 2017. PÁGINAS 151-173. ISSN 1851-6297 - ISSN 2362-3349 (EN LINEA) LA EDUCACIÓN COMO MECANISMO IGUALADOR DE OPORTUNIDADES: REEDITANDO VIEJAS DUdAS DESDE URUguay. PABLo LóPEZ Gómez

mujeres padecen menos el desempleo que los hombres, pero esa relativa ventaja va disminuyendo hasta invertirse en el grupo de mayor educación. Si se observa Latinoamérica como continente, no hay grandes diferencias entre los grupos excepto en la categoría 10 a 12 años (ciclo básico completo y todo o algún año del bachillerato) que es el grupo donde se registra mayor desigualdad. En resumen: la observación de este indicador no permite afirmar que la situación relativa de las mujeres frente a los hombres de su mismo nivel educativo varíe a medida que se educan más. Desde el punto de vista de la igualdad de oportunidades, el efecto de la educación sobre las diferencias de género no muestra una tendencia claramente establecida en el continente.

Otro indicador habitual respecto de la desigualdad de género es el referido a la brecha salarial, es decir la distancia de los ingresos laborales promedio percibidos por las mujeres y los varones activos. A diferencia de lo que ocurría con el desempleo, en este indicador todos los países muestran una brecha que favorece a los varones. Como se observa en la siguiente gráfica, Perú es el país donde esa diferencia es más notoria y Venezuela el más paritario. En Uruguay una mujer percibe promedio 83,6 pesos por cada 100 que recibe un hombre. En Chile y Brasil 80 y 81 respectivamente y Argentina es el país más paritario de la subregión con 89,2 . En el continente la brecha promedio es de 87,7 cada 100 .

\section{Gráfica 5: Brecha Salarial entre hombres y mujeres. Países de}

Latinoamérica 2011 o último dato disponible.

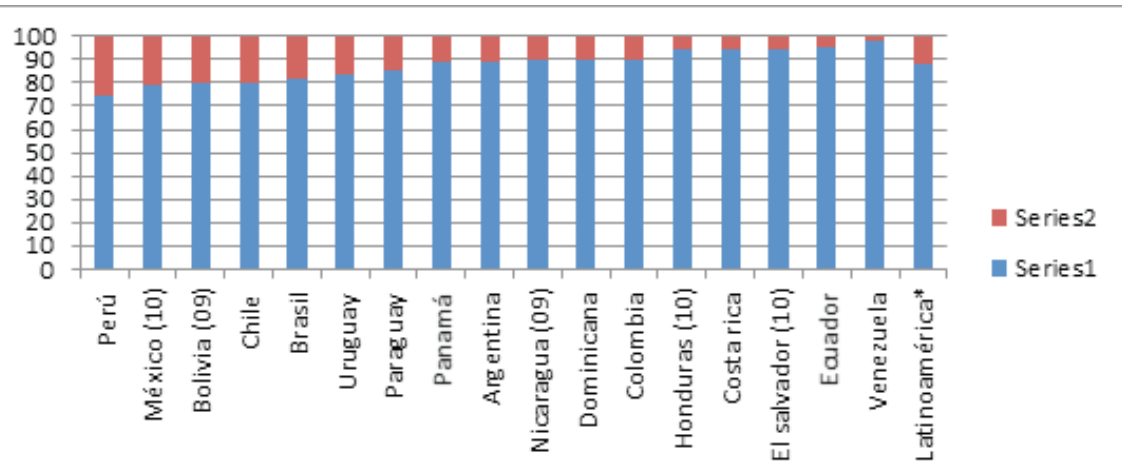

Fuente: elaboración propia en base a Cepalstat (consultado en 2013). *Promedio simple. 
Al igual que en el indicador anterior, la desigualdad de oportunidades debería disminuir a medida que aumentan los años de estudio. Vale decir, la brecha entre un hombre y una mujer menos educados, debería ser mayor que la de un par más educado. De esa forma, se podría intuir un efecto igualador de la educación en la estructura de oportunidades disminuyendo el del factor exógeno sexo. En la siguiente gráfica puede apreciarse que esto tampoco se confirma en este indicador.

\section{Gráfica 6: Brecha Salarial entre hombres y mujeres según años de}

estudio. Países de Latinoamérica 2011 o último dato disponible.

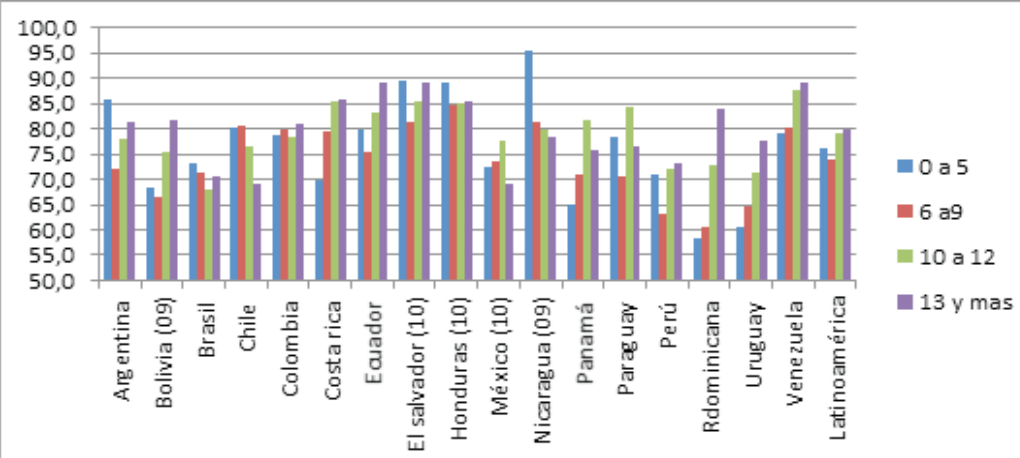

Fuente: elaboración propia en base a Cepalstat (consultado en 2013). *Promedio simple.

Si se mira el promedio del continente, puede afirmarse que la menor brecha se registra en las mujeres más educadas, sin embargo, la mayor brecha no corresponde al grupo de las menos educadas sino a una categoría intermedia. Asimismo, hay países como Chile y Nicaragua donde la tendencia es exactamente la inversa, a mayor educación la brecha aumenta. También hay países como Colombia y Honduras donde se observan pocas diferencias entre los distintos niveles educativos. En este caso Uruguay, a primera vista, se comporta de acuerdo a lo esperado respecto del rol de la educación en la igualdad de oportunidades, a mayor educación, menor es la brecha. Sin embargo, si se lleva los años de estudio más allá de los 13 años el comportamiento es otro. Según el informe 2013 de Inmujeres, para mujeres con 7 a 9 años de estudio la brecha es de 73 pesos cada 100, entre 10 y 12 es de $74,5 \%$, entre 13 y 15 es de $76,2 \%$. Hasta aquí se comporta en escalera ascendente, pero es interesante observar el siguiente grupo educativo, 16 y más años donde las mujeres ganan promedio el $67 \%$ de lo que gana un varón (La Buonora et al, 2013). Es decir, a partir del nivel licenciatura terminada y más, la brecha se intensifica siendo más importante que en todos los demás agrupamientos. Más allá de esta particularidad uruguaya, que tampoco puede considerarse una tendencia 
Revista de la Escuela de Ciencias de la Educación, año 13, nRo. 12, vol. 1, enero a junio de 2017. PÁGINAS 151-173. ISSN 1851-6297 - ISSN 2362-3349 (EN LíneA) La EdUCACIÓN COMO MECANISMO IGUALADOR DE OPORTUNIDADES: REEDITANDO VIEJAS DUdAS DESDE URUguay. PABLo LóPEZ Gómez

general del indicador, lo cierto es que -con estos datos- no se puede afirmar para la región que un mayor nivel educativo tenga un efecto igualador en las oportunidades salariales de hombres y mujeres.

\section{Igualdad de oportunidades y origen étnico}

El análisis de la desigualdad, que tiene como origen la pertenencia a una determinada etnia, también tiene una larga historia vinculada a distintos episodios de dominación y conquista de la humanidad. No obstante, desde el punto de vista estadístico, es relativamente incipiente la elaboración de indicadores que permitan utilizar la pertenencia a una etnia específica como analizador de determinadas condiciones sociales.

En Uruguay recién en el año 2006, en el marco de la Encuesta Nacional de Hogares Ampliada, se incluye una pregunta específica para la autoidentificación de la pertenencia étnica. Una pregunta similar se incorpora por primera vez en un Censo Nacional en 2011. Otros países de América Latina han incorporado la dimensión étnica en sus estadísticas con anterioridad y sus datos pueden servir para comparar la situación de distintos grupos étnicos de la región (es el caso de Brasil, Bolivia, Ecuador, Perú y Venezuela).

En América Latina, habitualmente, se indagan las diferencias en dos grandes categorías para datos generales: afrodescendientes y población indígena. A la interna de los distintos países suelen haber distinciones entre diferentes etnias, puesto que no todas tienen el mismo desempeño y son distintas las características de su integración. En Uruguay, si bien recientemente se han producido datos sobre la ascendencia indígena, aún no son suficientes para realizar comparaciones sistemáticas en los resultados. Por tanto el análisis de la situación nacional se centrará en la población afro.

Para hacer una aproximación al efecto, que la educación podría estar teniendo en la "igualación del terreno de juego", entre la población general y las minorías étnicas, será necesario observar el desempeño de algunos indicadores de resultado cruzado con nivel educativo y origen étnico, un procedimiento análogo al realizado con la variable sexo, pero con las limitaciones de una menor disponibilidad de datos. Lamentablemente los datos encontrados sólo permiten que realizar este ejercicio para el ingreso laboral.

En el informe de la OIT (2007), respecto a la situación laboral de las minorías étnicas en la región, se incluye la siguiente gráfica donde puede observarse el ingreso laboral por hora según origen étnico, de acuerdo a los años de educación cursados. El panorama es similar a lo que se observó respecto del sexo: no es regular la tendencia a que la brecha disminuya a medida que aumentan los años de estudio. De hecho, en varios países, incluyendo Uruguay, la brecha aumenta junto con el nivel educativo. Para las personas que tienen primaria completa o menos, la brecha es de apenas $4 \%$. En cambio, cuando se analizan las personas con 13 y más años de estudio, la brecha aumenta 
Revista de la Escuela de Ciencias de la Edudcación, año 13, nRo. 12, vol. 1, enero a Junio de 2017. PÁgINAS 151-173. ISSN 1851-6297 - ISSN 2362-3349 (EN LíNEA) LA EDUCACIÓN COMO MECANISMO IGUALADOR DE OPORTUNIDADES: REEDITANDO VIEJAS DUdAS DESDE URUguay. Pablo López Gómez

a un $29 \%$. Es decir, a medida que aumenta el logro educativo, las diferencias que podrían ser atribuidas a la desigualdad de oportunidades de origen racial también aumentan.

Gráfica 7: Proporción de ingreso laboral por hora de asalariados indígenas y afrodescendientes respecto de asalariados no indígenas ni afrosdescendientes, por años de escolaridad. América Latina, 6 países, alrededor del 2000.

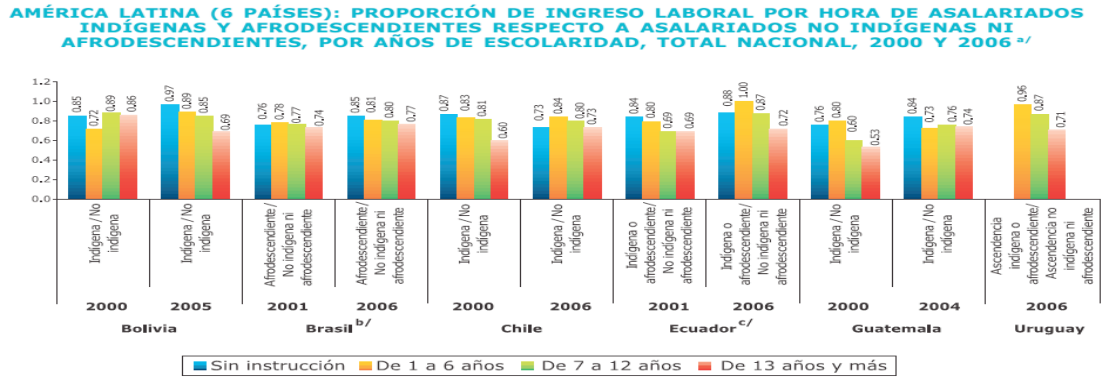

Fuente: OIT (2007, p. 48).

La situación de Uruguay se constata también en Bolivia y Brasil y tiene la misma tendencia en Ecuador, si no se toma en cuenta el grupo "sin instrucción". En Chile y Guatemala la brecha se mantiene más estable, en tanto los niveles educativos varían menos las diferencias de ingresos. En ninguno de los países, el hecho de tener mayores logros educativos implica, para las minorías étnicas, una disminución de las diferencias respecto del resto de la población.

Las siguientes tres gráficas reflejan las diferencias en los ingresos laborales de acuerdo a origen étnico y años de estudio en tres países. En los primeros dos, Brasil y Ecuador, se refiere a la población afrodescendiente. La tercera es de Guatemala y señala la desigualdad que padecen los pueblos indígenas. En todas se incluye la variable sexo.

Los comportamientos de las tres gráficas tienen similitudes y diferencias. En las tres, la brecha más importante en el ingreso laboral se acentúa especialmente en el grupo más educado, consecuente con la gráfica de brecha de ingresos anterior. En los tres países esa brecha es mucho menos significativa hasta los 11 años de estudio, pero con comportamientos distintos: mientras que en Brasil y Guatemala los ingresos de todos los grupos tienden a crecer con la educación, en Ecuador los ingresos de los menos educados son mayores que los ingresos de los que tienen 4 a7 y 8 a 11 años de estudio. En Brasil, el ingreso de las mujeres blancas y de los hombres afro tiene una evolución 


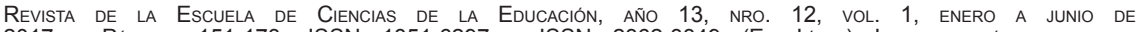
2017. PÁGINAS 151-173. ISSN 1851-6297 - ISSN 2362-3349 (EN LINEA) LA EDUCACIÓN COMO MECANISMO IGUALAdOR DE OPORTUNIDADES: REEDITANDO VIEJAS DUdAS DESDE URUGUay. PABLO López Gómez

similar, a medida que aumentan los años de estudios, intensificando su brecha respecto de los varones blancos durante toda la gráfica. En cambio, en Ecuador, la pertenencia a una minoría étnica resulta más determinante sobre los ingresos y tanto las mujeres como los varones blancos tienen mayores ingresos que mujeres y varones negros, conservando igual una brecha de género en la población blanca. Esto mismo sucede en Guatemala. Las gráficas confirman, además, la repetida cuestión de la interseccionalidad de las discriminaciones: en los tres países las mujeres de minorías étnicas son, en casi todos los tramos, las que perciben menos ingresos. En Brasil esto se intensifica a medida que aumenta la educación.

\section{Gráfica 8: Ingreso laboral promedio por condición étnica, sexo y tramo} educativo. Ecuador, Brasil (2006) y Guatemala (2000).

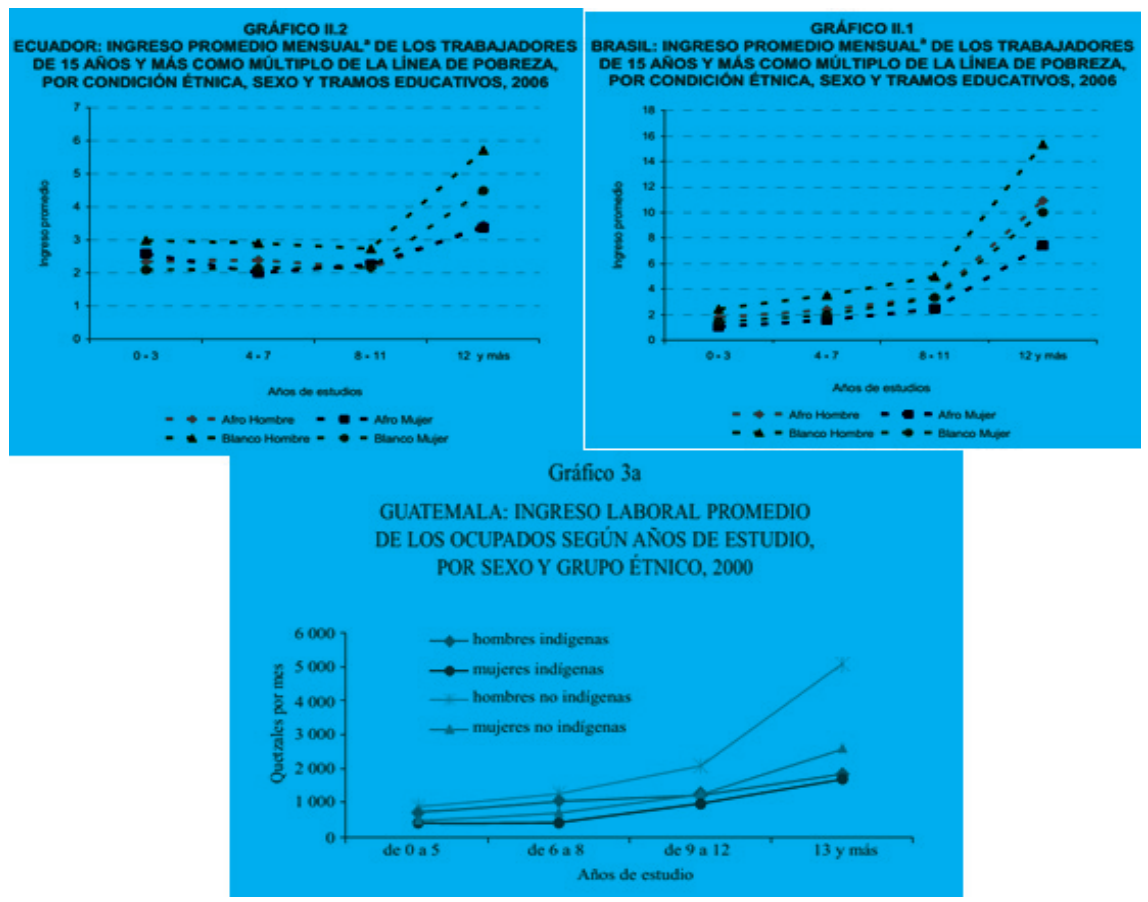

\section{Fuentes:}

Bello y Paixao (2008, pp. 40 y 42).

Valenzuela y Rangel (2004, p. 164).

Para el caso de Uruguay se elaboró una gráfica similar a las anteriores en base a la información de MIDES - INMUJERES (2008). El comportamiento del 
país es similar al de Brasil. En el grupo más educado los varones de las dos etnias ganan más que las mujeres, pero los salarios de las mujeres blancas y los varones afro son prácticamente iguales. La brecha principal está en la combinación de género y etnia, y va aumentando a medida que avanzan los niveles educativos. Por otro lado, las mujeres pertenecientes a las minorías son las peores remuneradas en todos los grupos educativos y además la brecha salarial va creciendo a medida que aumentan los años de estudio. En principio, la evidencia parecería desmentir la idea de que un mayor nivel educativo empareja el terreno de juego, disminuyendo el efecto de factores como el género o la pertenencia étnica, por lo menos en lo referido a los ingresos laborales.

Gráfica 9: Ingreso medio por hora de trabajo en población afrodescendiente y no afrodescendiente según nivel educativo y sexo.

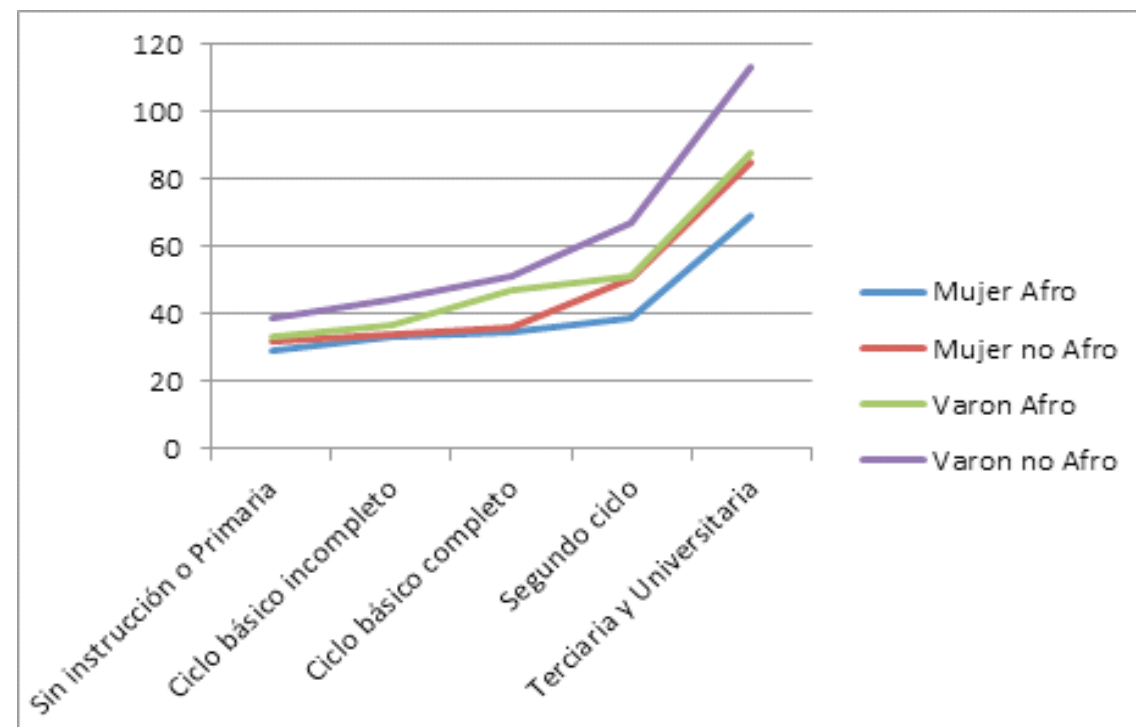

Fuente: Sistema de Información de Género de Inmujeres en base a ECHINE 2008.

\section{Limitaciones normativas de la igualdad de oportunidades}

Se ha presentado hasta aquí algunas ideas centrales de los teóricos de la igualdad de oportunidades. Sobre el final, es pertinente presentar algunas de las críticas que se les formulan a estas teorías. En 2012, François Dubet escribe un trabajo llamado "Repensar la justicia social", donde plantea que, hoy día, preponderan dos nociones de justicia social principales: la que se 
ocupa de la igualdad de posiciones o lugares y la que considera fundamental la igualdad de oportunidades.

Señala, desde la introducción, que considera ambas ideas de justicia son excelentes y que las dos ideas deben convivir en cualquier sociedad democrática. Sin embargo, esto no significa que los grupos de intereses que representan una y otra no sean diferentes, ni esconde que son diferentes sus motivaciones y formas de manifestarse. Asimismo, subraya que esta complementariedad no implica que no se deba establecer algún nivel de prioridad entre ellas.

Una sociedad no se percibe y no actúa de la misma manera según se incline por la igualdad de posiciones o la igualdad de oportunidades. En particular, los actores a cargo de la reforma social -los partidos de izquierda, en especial- se ven enfrentados a una elección que no pueden eludir eternamente. (Dubet, 2012, p. 14).

Las primeras críticas del autor se centran en estadísticas similares a las presentadas a lo largo del capítulo y apuntan, fundamentalmente, a señalar como el principio de Rawls (1995) de la diferencia (aquel que postula que, en la igualdad de oportunidades, las desigualdades engendradas por la competencia no deben perjudicar a los más desprotegidos) no se cumple. El autor señala que un sistema de justicia basado en la igualdad de oportunidades -como el estadounidense- ha aumentado la desigualdad en la distribución, por un lado, pero también la pobreza en términos absolutos.

Dubet explica este fenómeno debido a la indiferencia hacia estos resultados que se produce en la retórica de la igualdad de oportunidades, que siempre observa sus resultados en términos de logros: cuántas mujeres acceden al gobierno, cuántos estudiantes pobres van a colegios de elite, etc.

La cuestión de la representatividad se hace clave: si tenemos $50 \%$ de mujeres en el gobierno, siendo $50 \%$ de la población, y además los hijos de los sectores más vulnerados que representan el $15 \%$ de la población (por ejemplo), llegan a buenos colegios y representan un $15 \%$ de la matrícula, podríamos pensar que hay igualdad de oportunidades. Sin embargo, a los colegios de élite asisten unos miles de alumnos, pero el " $15 \%$ de niños de sectores vulnerables," representa millones de pobres que no dejan de serlo por esta representación equitativa. Así, la igualdad de oportunidades, por su manera de ver y medir, ilumina los logros y se desentiende de los resultados de quienes no alcanzan esos logros. 


\section{Conclusiones}

Como se expresó desde un comienzo, este artículo no pretende desmentir ciertas correlaciones positivas entre el nivel educativo logrado y el acceso a bienes sociales que, como se observa en los datos presentados en el apartado sobre etnia (gráficas 8 y 9), se constata, principalmente a partir del nivel terciario.

No obstante, se observa que el rol que le otorga a la educación la teoría de la igualdad de oportunidades -que la coloca como la "niveladora del terreno de juego", permitiéndole a personas de grupos sociales en desventaja igualar sus posibilidades de acceso al bienestar- no se confirma de forma universal ni de manera homogénea.

En el apartado sobre género se observa un cierto efecto positivo del nivel educativo en la posibilidad de formar parte de la población activa, que podría estar seriamente sesgado por el efecto de este indicador sobre la paridez. En los demás indicadores revisados, desempleo y brecha salarial, el efecto es muy desparejo en los países de la región (aunque con una cierta tendencia contraria a la esperada). En especial, respecto a la diferencia en los salarios, con altos niveles educativos la brecha entre hombres y mujeres tiende a aumentar. En lo que refiere a etnia, donde sólo se analizó brecha salarial, la tendencia es la misma, aumentando las diferencias a medida que aumentan los niveles educativos alcanzados, tanto para la población afro como para la población indígena.

Finalmente, más allá de las corroboraciones empíricas, cabe cuestionarse la pertinencia de tener la igualdad de oportunidades como horizonte de las políticas educativas, por las discusiones normativas que pueden oponérsele a esta teoría de justicia.

\section{Referencias Bibliográficas}

- Antón, J., Bello, A.; Del Popolo, F.; Paixão, M., \& Rangel, M. (2009). Afrodescendientes en América Latina y el Caribe: del reconocimiento estadístico a la realización de derechos. Santiago de Chile: Naciones Unidas.

- Arneson, R. (1989). Equality and Equal Opportunity for Welfare. Philosophical Studies: An International Journal for Philosophy in the Analytic Tradition, 56 (1), 77-93.

- Bello, Á. y. (2008). Estado actual del cumplimiento de los Derechos Civiles, Políticos, Económicos, Sociales y Culturales de la Población Afrodescendiente en América Latina. Santiago de Chile: CEPAL.

- Bourdieu, P. (2003). Cuestiones de sociología. Madrid: Istmo.

- CEPAL. (2007). Panorama social de América Latina. Santiago de Chile: CEPAL.

- Dubet, F. (2011). Repensar la justicia social contra el mito de la igualdad de oportunidades. Buenos Aires: Siglo XXI.

- Dworkin, R. (2000). Sovereign Virtue: The Theory and Practice of Equality. Harvard University Press: Harvard

- Ferrerira, F. H. G. y Walton, M. (2005): La trampa de la desigualdad. Por qué la equidad 
debe ser un componente esencial de la política de desarrollo, Finanzas y Desarrollo, 1(12), 34-38

- La Buonora, L.; Pandolfi, J.; Pieri, D.; Semblat, F., Vargas, X. \& Villamil, L. (2013). Estadísticas de género 2013. Evolución de los indicadores de género en el período 20092013. Montevideo: Inmujeres.

- Lopez, A. \& Varela, C. (2016). Maternidad en adolescentes y desigualdad social en Uruguay. Montevideo : UNFPA - UDELAR .

- $\quad$ Paes de Barros, R.; Ferreira, F.; Molinas, J.; Vega, J. \& Saavedra, C. (2008). Midiendo la desigualdad de oportunidades en América Latina y el Caribe. Washington: Banco Mundial.

- Pateman, C. (1995). El contrato Sexual. Barcelona: Anthropos.

- Puyol, A. (2010). El sueño de la igualdad de oportunidades: Crítica de la ideología meritocrática. Bacelona: Gedisa.

- Rawls, J. (1995). Teoría de la Justicia. México: FCE.

- Roemer, J. (1998). Equality of Opportunity. Cambridge, Mass.: Harvard University.

- Sen, A. (2000). Social Justice and Distribution of Income. En B. Atkinson, \& F. Bourguignon, Handbook of Income Distribution (pp. 59-86). Amsterdam: North Holland.

- Valenzuela, M., \& Rangel, M. (2004). Desigualdades entrecruzadas. Pobreza, género, etnia y raza en América Latina. Santiago de Chile : OIT .

- Varela Petito, C., Pollero, R., \& Fostik, A. (2008). La fecundidad: evolución y diferenciales en el comportamiento reproductivo. En Varela Petito, C. Demografía de una sociedad en transición. La población uruguaya a inicios del siglo XXI (pp. 35-69). Montevideo: Trilce. 